\title{
In-Vivo Connectivity in Monkeys
}

\author{
Wim Vanduffel
}

\begin{abstract}
Major efforts are underway to provide highly detailed descriptions of static anatomical brain connectivity in rodents, even down to the level of individual synapses. To fully understand brain functioning and to bridge the gap between rodents and humans, however, I argue in this chapter that effective connectivity studies in nonhuman primates are equally critical. The primate community should embrace the novel, high-precision genetic-based toolkits developed in invertebrates and rodents to study how activity in one brain region influences that in connected brain regions. These methods will allow us to measure true functional weights of anatomical connections during highly varying cognitive and perceptual demands in primates. Why monkeys, and why effective connectivity in addition to anatomical connectivity? First, the nonhuman primate is critically important to understand the functioning of the human brain since important brain regions, such as the granular prefrontal cortex carrying higher cognitive functions, are lacking in rodents as opposed to primates. Second, a pure anatomical description of connections at different scales may be useful to constrain models of brain functioning, however, it has little value to explain perception and behavior emerging from dynamic neuronal activity in distributed brain networks. Hence, tools that allow us to measure these dynamics at large scale and to causally interfere with the system at high temporal and spatial resolution are required to increase our understanding of changes in information processing at different stages within a functional network. In this chapter I will review past and emerging methods to study effective connectivity (mainly) in nonhuman primates, in other words how activity within a given brain area influences processing in anatomically connected brain regions. I will also argue that high-resolution whole brain imaging in monkeys may be invaluable to guide reversible perturbations and massive neurophysiological recordings simultaneously within multiple nodes of functional networks.
\end{abstract}

\footnotetext{
W. Vanduffel $(\bowtie)$

Department of Neurosciences, Laboratory for Neuro-and Psychophysiology, KU Leuven Medical School, 3000 Leuven, Belgium
}

Harvard Medical School, Boston, MA 02115, USA

Martinos Center for Biomedical Imaging, Massachusetts General Hospital, Charlestown, MA 02129, USA

e-mail: wim@nmr.mgh.harvard.edu 


\section{Anatomical, Functional and Effective Connectivity in Animal Models}

Perception and behavior emerges from the concerted activity of millions of neurons constituting a distributed but connected network of brain regions. To understand brain function, it will not suffice to define the detailed characteristics of single neurons or of a handful of "representative" neurons in one or a few areas simultaneously. A critical piece of information required for building biologically-plausible models of brain function is the manner in which these neurons are connected anatomically. Following decreasing interest in connectivity studies over several decades, this field has regained considerable impetus in recent years, mainly thanks to the appearance of new technologies allowing high throughput of connectivity data, which has been fueled by high-profile funding schemes such as the Connectome (Van Essen et al. 2012) and BRAIN (Devor et al. 2013) initiatives in the USA, the Human Brain Project (Markram 2012) in Europe and the BRAIN/ MINDS (Okano et al. 2015) project in Japan. Also privately-sponsored institutes such as the Alan Institute and Janelia farms have launched projects aimed at collecting large-scale connectomics data, mainly in rodents. The resulting static descriptions of anatomical connections will be of great benefit for constraining models of cortical functioning at the micro-, meso- and macroscale.

However, critically important information, that cannot be gleaned from static descriptions of anatomical connectivity, concerns the variations in the functional strength of these connections across the highly-dynamic, constantly-varying states of the subject. Although intuitively one might expect that a strong anatomical connection exerts more weight on a target area than a weaker one, it actually depends on a variety of factors including the subject's behavioral state, the specific perceptual or task demands, the type of connection (i.e. feedforward or feedback), the excitation/inhibition balance, and the neurotransmitter systems involved. Therefore, the extent to which a set of neurons can influence processing in another set of neurons under various mental states and behavioral conditions constitutes crucial information required to fully understand brain function. In general, this is referred to as effective connectivity as opposed to functional connectivity (Friston 2011). Measures of functional connectivity are typically based on correlations of neuronal or hemodynamic activity across brain sites. Effective connectivity, on the other hand, relates to the mechanisms driving such correlated activity. This can be caused by common inputs, or by regions that actively influence activities in connected areas. Effective connectivity can be inferred statistically by, for example, using Granger causality, which relies on the fact that the history of activity in a source region is more predictive of activity in a target region than the history of the latter region itself. However, the only direct method to assess whether a particular region has a causal effect on processing in other regions is the use of focal perturbation methods in combination with a read-out tool such as electrophysiology, or any kind 
of functional imaging. In this chapter I will briefly discuss several methods that are being used to investigate effective connectivity in awake animal models, thereby focusing on the past and current work of my group and speculating about future approaches.

\section{Towards Causality: Effective Connectivity with Focal Perturbation Tools}

The oldest perturbation tool is a simple lesion. The major disadvantage here is the irreversible nature of lesions, rendering test-retest experiments impossible. Moreover, the permanent nature of a lesion can trigger compensatory brain mechanisms whereby lost functionality at the site of the lesion may be partially assumed by other regions, although the temporal dynamics by which compensatory mechanisms are engaged remain unknown. Reversible perturbation methods are obviously advantageous compared to lesions.

Traditional focal, reversible perturbation tools used in animals include chemical, thermal, and electrical methods. Less focal, though non-invasive, inactivation protocols include transcranial magnetic stimulation (Gerits et al. 2011), and transcranial pulsed ultrasound methods (Tufail et al. 2010). Frequently-used chemical inactivation methods rely on the injection of local anesthetics or GABAagonists such as muscimol (Hikosaka and Wurtz 1985). These chemicals have a relatively long half-life when injected, rendering them less than optimal for fast test-retest protocols and leaving them vulnerable to possible rapid functional reorganizations of the brain. Focal cooling of the brain is quite appealing, since the activity of neurons under the cooling probe can be blocked when the temperature falls below $20^{\circ} \mathrm{C}$ without affecting transmission in traversing fibers (Lomber et al. 1994; Vanduffel et al. 1997; Lomber et al. 1999). Although several cycles of cooling can be performed within an experimental session, a major issue is that cooling is applicable mainly to easily accessible structures at the cortical surface. Cooling subcortical structures, or within a sulcus, without damaging tissue is technically demanding and affects neighboring tissue (such as both banks of a sulcus). Almost two decades ago, we cooled extrastriate cortex in awake cats in combination with deoxyglucose measurements, through which metabolic activity can be assessed throughout the entire brain (Vanduffel et al. 1997). As predicted by the excitatory nature of most cortico-cortical connections, we observed reduced metabolic activity in areas connected with the cooled region. Intriguingly, we observed that the effect on feedforward connections was stronger than predicted based on the anatomical strength of these connections alone, and that the converse was true for feedback connections. To the best of my knowledge, this was the first experimental evidence that the strength of an anatomical connection does not predict its functional weight (see Fig. 1). 

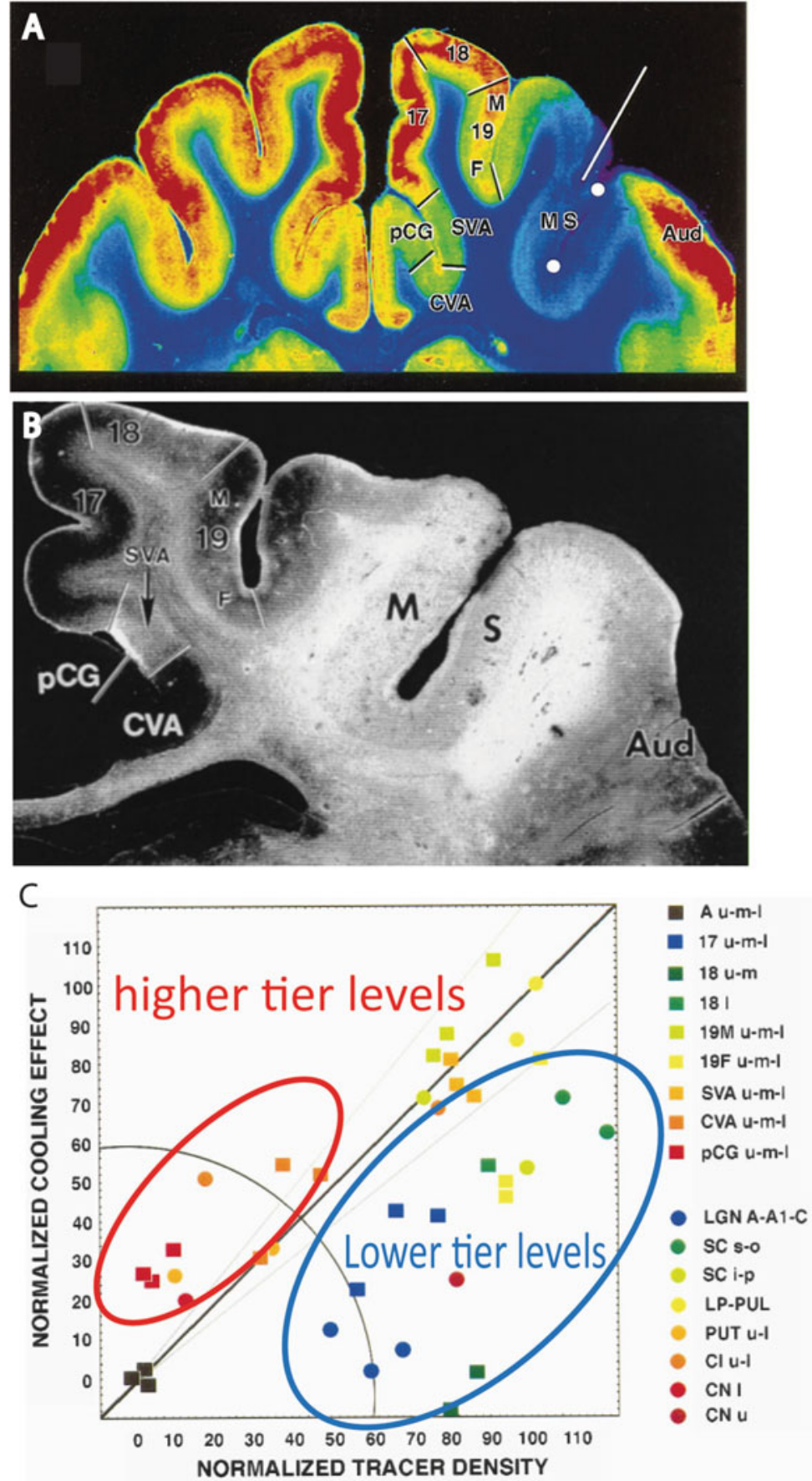

Fig. 1 (a) Color-coded image of 2-Deoxyglucose (2DG) concentrations in a coronal section of the cerebral cortex of the cat during cooling-induced deactivation of the middle suprasylvian sulcus (MS). White circles in MS sulcus represent position of the cooling probe. Color scale indicates 
Electrical microstimulation is a focal perturbation tool used to map sensory cortex in humans (Rasmussen and Penfield 1947). Despite its high temporal resolution, microstimulation of local neuronal activity can be facilitatory or inhibitory, depending on parameters such as the amplitude, frequency, and duration of the stimulation trains (Tehovnik and Lee 1993). Another drawback relates to the microstimulation currents that can produce electrical artifacts during and immediately after the pulses when the procedure is combined with electrophysiology (Premereur et al. 2012, 2014; Moore and Armstrong 2003). Also, as nicely illustrated by Clay Reid's group, it is not always obvious which neuronal elements are affected at the electrode tip (Histed et al. 2009).

More recently, genetically-based perturbation tools with superior temporal and spatial resolution have emerged, the most established of these being optogenetics (Yizhar et al. 2011). This method enables one to manipulate activity of neurons on a millisecond time-scale. Optogenetics relies on light-sensitive proteins (opsins) incorporated into neuronal membranes using viral vectors or genetically engineered organisms [for details see Karl Deisseroth's (2015b)]. The activities of cells expressing the opsins can be manipulated by illumination with specific wavelengths. Depending on the opsin type, neurons and other cells can be depolarized (activated) or hyperpolarized (deactivated) when illuminated. The appeal of optogenetics is its millisecond temporal resolution and its superior spatial resolution relative to other techniques (Deisseroth 2015a). Although optogenetics allows modulation of activity in specific cell types, the results of cell-type specific optogenetics should be interpreted with some caution, because information arising from transduced cells is immediately transmitted to downstream neurons within the targeted micro-and macro circuitry. Therefore, any readout method (including behavior) with a temporal resolution lower than tens of milliseconds will encounter difficulties in differentiating between effects induced by the modulated cells and those (in)directly connected. Immediate downstream effects have been nicely demonstrated in monkeys by Bob Desimone's group, who observed, exactly as expected, enhanced neuronal activity, consequent to transduction with a depolarizing opsin, near the targeted site (Han et al. 2009). However, they also measured consistent suppressed neuronal activity in the site injected with the viral

Fig. 1 (continued) 2DG concentrations. White scale bar, $5 \mathrm{~mm}$. SVA splenial visual area, Aud auditory cortex, $L G N$ lateral geniculate nucleus, $P u l$ pulvinar nucleus, $L P$ lateral posterior nucleus; 17,18 areas 17 and 18; 19M, 19F area 19 medial or fundal parts. (b) Distribution of $[3 \mathrm{H}]$ proline and $[3 \mathrm{H}]$ leucine transported after injections in the middle suprasylvian suclus. Darkfield illumination indicating strength of connections from MS sulcus. (c) Normalized tracer density versus normalized cooling effect on 2DG concentration for a variety of cortical and subcortical structures. Solid line represents slope of unity where tracer density and effects on 2DG uptake are equal. Gray lines represent $20 \%$ deviation from these values. Squares indicate cortical structures, circles subcortical structures. The color-coded labels for the various structures reflect levels of visual processing: blue, early; green yellow, intermediate; and red, late. Points below the diagonal show a weaker 2DG effect than anticipated from the anatomy, whereas points above the diagonal show a stronger 2DG effect than anticipated. Figure adapted from Vanduffel et al. (1997) 
vector construct. The latter effect appeared at longer latencies, thus in neurons that are at least one synapse away from the transduced neurons.

Other very promising genetically-based methods include DREADDs (designer receptors exclusively activated by designer drug), whereby an artificial receptor is expressed in the cell membranes which can be activated by an artificial ligand (Dong et al. 2010). Hence, this method is much less invasive than optogenetics since no optical fiber is required, only the administration of a drug that activates the receptor. These DREADD-type approaches are very promising, especially for applications where the temporal resolution of the perturbation is less important.

Ultimately, to completely understand the neuronal processes underlying perception and behavior, one will need to acquire electrophysiological signals at the single-cell level simultaneously from all brain sites involved in these processes. Furthermore, to clarify whether all such neuronal populations are critically involved, their activity needs to be up-or down regulated while the effect on perception or behavior is being assessed. Despite recent technological advances for recording from several dozens of single neurons simultaneously, even in multiple areas, and despite the astounding innovations in genetically-based methods to reversibly perturb activity in brain cells, this ambitious goal cannot be achieved as of yet, especially in primates. To make this daunting task somewhat tractable and to vastly reduce the needle-in-a-haystack aspect, I propose that targeting of these multiple recording sites should be guided by high-resolution whole-brain imaging (Vanduffel et al. 2014). We recently developed implanted phased-array coils in monkeys to obtain sub-millimeter whole-brain functional data with standard clinical MR scanners, which might serve this purpose (Janssens et al. 2012) (see Fig. 2).

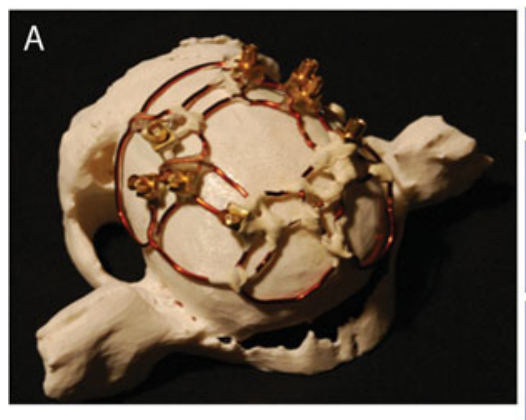

1Ch external $4 \mathrm{Ch}$ external $8 \mathrm{Ch}$ external $8 \mathrm{Ch}$ implant

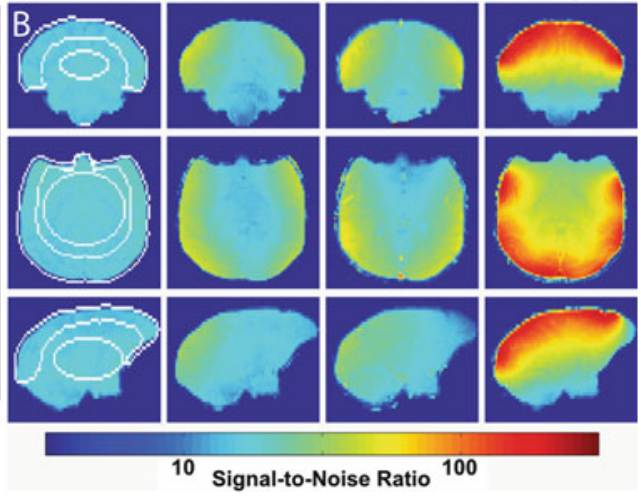

Fig. 2 (a) Photo of phased-array MR coil embedded in the headset of the monkeys (b) Increase in signal-to-noise ratio of implanted phased-array coil relative to other external phased-array coils. This coil allows sub-millimeter fMRI in monkeys at $3 \mathrm{~T}$. Figure adapted from Janssens et al. (2012) 


\section{Effective Connectivity: Combined Electrical Microstimulation and Whole-Brain fMRI}

In the past, we have employed whole-brain imaging during microstimulation of the FEF (FEF-EM), a region that can be behaviorally-defined, based on its involvement in saccade generation. When this region is electrically stimulated with low currents, monkeys make eye movements whose direction and amplitude depend on the exact location of the electrodes within the topographically-organized FEF. The endpoints of these saccade vectors correspond to the movement fields of the neurons surrounding the electrode tip. Mounting evidence suggests that, in addition to its functional role in generating eye movements, the FEF is an area sending selective top-down signals to occipital regions in order to modulate incoming sensory activity in an attention-dependent manner. FEF is well-positioned for the task, since it receives connections from several higher-order neighboring areas involved in cognitive control (Schall et al. 1995). Moreover, it comprises a topographic map representing the visual field in a relatively well-organized manner (Janssens et al. 2014), hence FEF has the capacity to send spatially-specific signals back to upstream areas (Schall 1997).

Electrophysiology has shown that when FEF is stimulated at current levels insufficient to generate a saccade, activity in single V4 (Moore and Armstrong 2003) and LIP (Premereur et al. 2014) neurons can be enhanced for stimuli placed in the stimulated movement fields - as opposed to stimuli shown outside these movement fields. Furthermore, the modulation of the V4 firing rates is more pronounced when competing stimuli are shown, mimicking distractors surrounding a target during a spatial attention experiment. These sorts of changes in neuronal firing rates resemble the selective spatial attention effects previously observed in V4 (McAdams and Maunsell 1999).

In a follow-up experiment, Tirin Moore's group also showed that this subthreshold electrical stimulation in FEF resulted in improved detection thresholds for low-contrast stimuli flashed in the FEF movement fields (Moore and Fallah 2004). Albeit indirect, the combination of the behavioral and electrophysiological results obtained in V4 provided strong evidence that FEF can influence neuronal activity in visual cortex, in a manner very similar to that observed during selective spatial attention. Indeed, selective attention to a specific location in the visual field will enhance the neuronal processing of stimuli presented at that location, and these effects are particularly pronounced for less salient stimuli and when distractor stimuli are present. A direct link between microstimulation results and selective attention remains unverified, however, since the behavioral and electrophysiology experiments were not performed concurrently. Hence, the existence of a direct link between enhanced V4 activity and improved perception cannot yet be confirmed.

To complement these two groups of experiments, we attempted to visualize how increased FEF activity modulates activity throughout the visual cortex, rather than at the single-neuron level, by combining FEF-EM with functional magnetic resonance imaging (fMRI) in monkeys (Ekstrom et al. 2008). We chose FEF as the 
(first) target in a series of combined EM-fMRI experiments, since the behavioral read-out, i.e. EM-triggered saccades, is straightforward. This is especially beneficial during fMRI experiments, since currents induced in the electrodes, by the switching gradient fields of the MR scanner, would have been immediately apparent in the animal's eye-movements. In fact, simulations prior to the experiments, taking into account the MRI sequences, properties of the gradient coils, as well as tissue and electrode properties, indicated that we barely induced currents in the electrodes. When FEF was stimulated with currents well below those necessary to evoke saccades, exactly as in Tirin Moore's experiments (Moore and Armstrong 2003), we could visualize the network of areas that are anatomically linked with the FEF using concurrent fMRI. The EM-evoked activity patterns over the entire brain matched surprisingly well those patterns of connectivity obtained using traditional tract-tracing methods for which animals need to be sacrificed (see Fig. 3). Hence the combination of electrical microstimulation with whole-brain fMRI is an excellent tool for revealing, at least as a proxy, in-vivo anatomical connectivity information (with some caveats, see below).

More interestingly, when a visual stimulus was placed in the stimulated FEF movement fields, we observed modulation of fMRI activity in those parts of the visual cortex that are driven by the visual stimulus. This modulation was

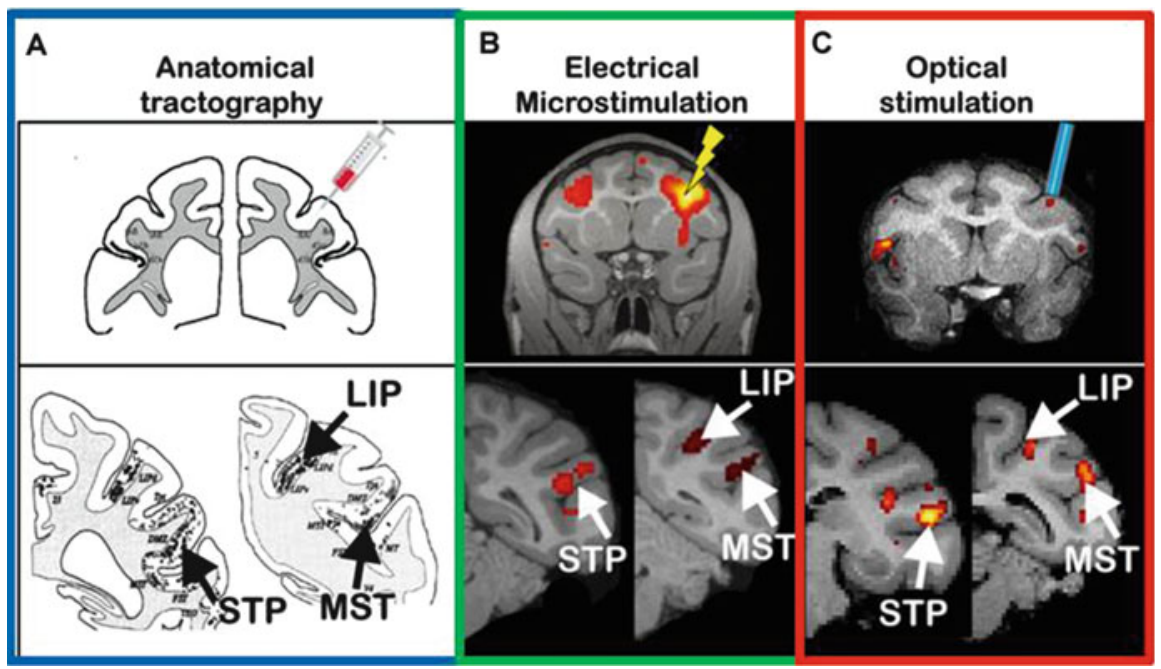

Fig. 3 Comparison between (a) anatomical tractography, (b) electrical microstimulation (EM), and (c) optical stimulation. Injections of tracer in the frontal eye fields (FEF) of macaque monkey produced labeled cells in the lateral intraparietal area (LIP), the medial superior temporal area (MST), and the superior temporal polysensory area (STP) (Schall et al. 1995). fMRI combined with EM of the FEF resulted in fMRI activations in LIP, MST, and STP [67] (Ekstrom et al. 2008). Monkey optogenetic-fMRI with channel-rhodopsin-transduced neurons in FEF also showed an increase in fMRI signal in LIP, MST, and STP (Gerits et al. 2012). Note the striking correspondence between the in-vivo microstimulation and optogenetic induced fMRI activations and the ex-vivo tractography data. Figure adapted from Gerits and Vanduffel (2013) 
topographically-specific to the representations of the stimuli placed within the FEF movement fields and included regions with both increased and decreased fMRI activity.

Thus, as in Tirin Moore's experiments, we found modulation of fMRI activity in visual cortex for stimuli presented in the stimulated FEF movement fields. A surprising discrepancy between the results of this EM-fMRI experiment and single-unit data from V4, however, is that we generally observed decreased activity in the same voxels of visual cortex that are optimally driven by visual stimuli in the absence of FEF microstimulation. In contrast, voxels that were only weakly driven by the visual stimulus showed enhanced fMRI activity when the corresponding FEF movement fields were electrically stimulated. This counterintuitive result can be explained, however, by the nature of the stimuli and the experimental design. In our first series of experiments, we used high-contrast, colored gratings presented one at a time. If electrical stimulation of specific FEF-movement fields would mimic 'covert attention' to that location, one could argue that the evoked modulatory effects in visual cortex would be small for a highly salient (high-contrast) stimulus, since no additional 'attentional boost' is required. The same holds true for stimuli presented in isolation without competing distractors, as attention effects are typically stronger for targets embedded in a field of distractors compared to targets in isolation.

In two follow-up experiments, we addressed whether FEF-EM would induce stronger modulatory effects in visual cortex for low-versus high-salient stimuli by manipulating either the luminance contrast or by adding 'distractor' stimuli (Ekstrom et al. 2009; Ekstrom et al. 2008). These experiments confirmed that FEF-EM had a much stronger effect on the low-contrast luminance-defined stimuli (see Fig. 4). Activity in visual cortex was upregulated for low-contrast gratings and unaffected, or even suppressed for high-contrast stimuli, thus confirming the results of the first experiment with the high-contrast colored gratings. In essence, we observed a contrast-gain as opposed to an activity-gain effect, whereby the contrast-response curves were shifted to the left. In the second control experiment, concurrent FEF-EM demonstrated that the representation of a grating embedded in a scene including several competing gratings showed much larger modulatory effects compared to a grating presented in isolation, keeping all other experimental parameters identical.

These control experiments showed that FEF is capable of modulating representations in visual cortex in a manner that closely resembles attention-dependent effects. Although the experiments were performed in the absence of explicit attentional requirements, the effects observed are surprisingly similar to those obtained during selective spatial attention (Reynolds and Heeger 2009). This indicates that the necessary hardware, i.e. wiring, neurotransmitter systems, and axonal properties, exist that allow the FEF to alter sensory-driven activity in visual cortex based on non-retinal signals such as selective attention. As we have stated, conclusive evidence for this hypothesis needs to be provided by studies in which the FEF is stimulated while behavioral and neuronal effects are recording simultaneously. 

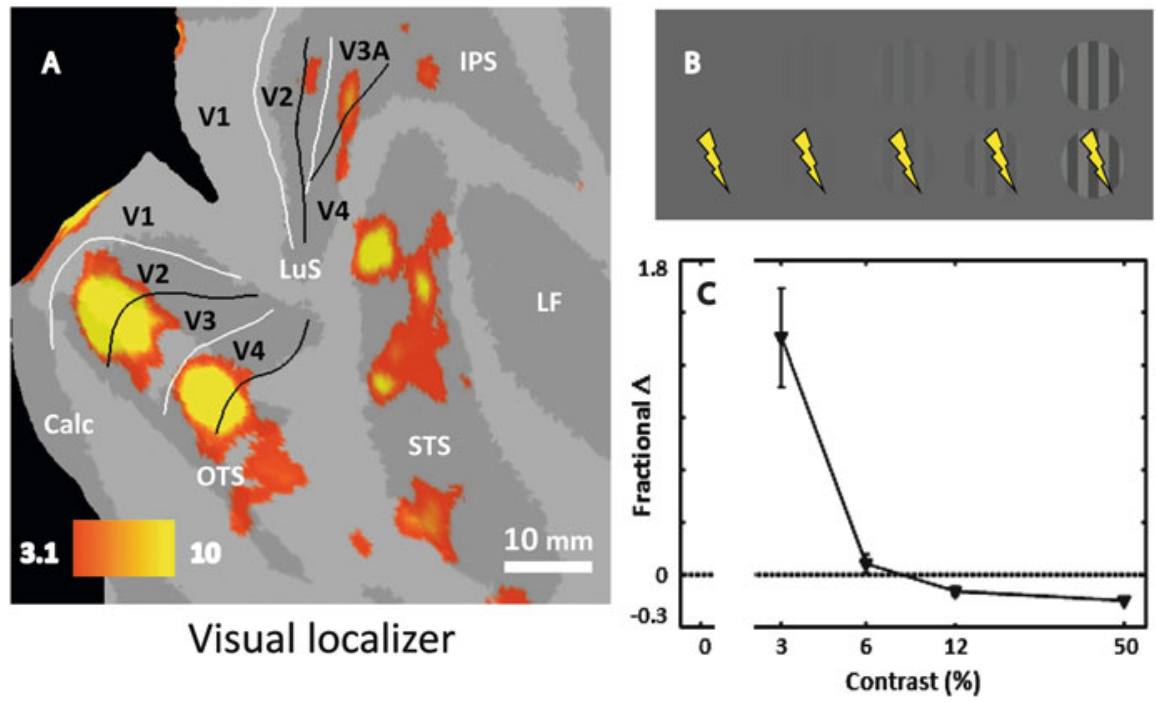

Fig. 4 Electrical microstimulation of monkey FEF boosts activity in visual cortex for low- but not high-contrast gratings. (a) Visually-driven activations by the stimuli on a flattened representation of occipital cortex. These voxels, obtained from the localizer experiment, were used to perform the analyses in panel (c). (b) Stimuli (contrast-varying gratings) used in the fMRI experiment. These were paired with and without concurrent electrical stimulation of the corresponding FEF movement fields. (c) Fractional differences in MR signal change in area V1, indicating the effective change in fMRI activity caused by FEF-EM relative to the visual-only activation level. In all panels, error bars indicate 1 SEM across epochs; some error bars are smaller than the symbol used. Note the large positive effect for low contrast and even a negative effect for high contrast stimuli. Figure adapted from Ekstrom et al. (2009)

\section{Novel Genetically-Based Perturbation Methods?}

A possible caveat in using electrical microstimulation pertains to the inherent difficulties in distinguishing between the ortho- and anti-dromic effects of EM. In other words, the EM effects observed in V4 neurons (Tirin Moore's studies) and in visual and parietal cortex in general (our studies) might be either the result of activation of the axon terminals of neurons with cell bodies in the visual cortex or, alternatively, of neurons located in the FEF that project to visual cortex. In lieu of FEF's proposed role as a source area sending higher-order cognitive signals to sensory cortex, the latter interpretation is the more favorable, yet conclusive evidence is largely lacking.

A possible solution to this conundrum is the use of focal pharmacological receptor (anta)gonists that specifically alter the output of the targeted area (Noudoost and Moore 2011). Alternatively, one could rely on optogenetics whereby, with the right combination of serotype and promoter in the viral vector construct, only projection cells are targeted, and no cells are retrogradely 
transduced. Even more appealing approaches include the use of viral vectors with highly-efficient retrograde transduction capacities. These can be injected in the projection sites of FEF after which the retrogradely-labeled cell bodies in the FEF can be targeted with optogenetics (Yizhar et al. 2011). Although such retrograde, or other conditional gene expression, approaches have been highly successful in rodents (Packer et al. 2013), there are no studies to date where retrogradely-driven optogenetics has been used in the cerebrum of nonhuman primates. A very elegant study using a highly efficient retrograde transduction system in nonhuman primates, resulting in exclusive expression of neurotoxin-producing enzymes in projection cells, has shown its efficacy in the spinal cord of monkeys (Kinoshita et al. 2012). Conceivably, similar pathway-specific, genetically-based perturbation methods will soon become applicable in the cerebrum of macaques. The enormous advantage of such techniques is that, instead of a specific region, the functional role of a specific pathway between two regions can be investigated.

A few years ago, we optogenetically targeted specific regions in frontal cortex of monkeys performing a saccade task, guided by prior fMRI maps (Gerits et al. 2012). Although optogenetically-induced neuronal effects in monkeys had been described previously (Han et al. 2009; Diester et al. 2011), our fMRI-guidance strategy allowed us to observe, for the first time, an optogenetically-induced behavioral effect in monkeys-i.e. faster saccades during an eye-movement task. When combined with concurrent fMRI, we also observed increased activity in connected regions, strongly resembling the anatomically-connected areas, and hence mimicking the electrical microstimulation effects described above (see Fig. 3). Meanwhile, several groups replicated optogenetically-induced behavioral effects in monkeys with different tasks and targeting different regions but without fMRI-guidance (Cavanaugh et al. 2012; Dai et al. 2014; Ohayon et al. 2013; Jazayeri et al. 2012). fMRI-based guidance, however, will greatly facilitate experiments aimed at simultaneously targeting multiple sites in the same subjects. With optogenetics, one can then temporarily activate or inactivate one or several of these nodes, even within specific subcomponents of a behavioral task.

In conclusion, I would argue that the genetically-based tools, which have recently instigated a revolution in rodent-based neuroscience, will ultimately find their way into nonhuman primate research. Although achieving cell-specificity will be challenging in monkeys due to the lack of Cre-lines, this animal model is crucial for elucidating the underpinnings of high-level perceptual and cognitive processes absent in rodents. To study multiple nodes within a functional network, highresolution functional imaging will greatly advance the precision of the targeting, be it with high-throughput electrodes, viral vector injections, or pharmacological agents.

Acknowledgements This work was supported by Inter-University Attraction Pole 7/11, the Research Foundation Flanders; KU Leuven (Programme Financing PFV/10/008), Hercules, and the Human Brain Project. 
Open Access This chapter is distributed under the terms of the Creative Commons AttributionNoncommercial 2.5 License (http://creativecommons.org/licenses/by-nc/2.5/) which permits any noncommercial use, distribution, and reproduction in any medium, provided the original author(s) and source are credited.

The images or other third party material in this chapter are included in the work's Creative Commons license, unless indicated otherwise in the credit line; if such material is not included in the work's Creative Commons license and the respective action is not permitted by statutory regulation, users will need to obtain permission from the license holder to duplicate, adapt or reproduce the material.

\section{References}

Cavanaugh J, Monosov IE, McAlonan K, Berman R, Smith MK, Cao V, Wang KH, Boyden ES, Wurtz RH (2012) Optogenetic inactivation modifies monkey visuomotor behavior. Neuron 76:901-907

Dai J, Brooks DI, Sheinberg DL (2014) Optogenetic and electrical microstimulation systematically bias visuospatial choice in primates. Curr Biol 24:63-69

Deisseroth K (2015a) Optogenetics: 10 years of microbial opsins in neuroscience. Nat Neurosci 18:1213-1225

Deisseroth K (2015b) Form meets function in the brain: observing the activity and structure of specific neural connections. In: Kennedy H, van Essen DC, Christen Y (eds) Micro-, meso- and macro- connectomics of the brain. Springer, Heidelberg

Devor A et al (2013) The challenge of connecting the dots in the B.R.A.I.N. Neuron 80:270-274

Diester I, Kaufman MT, Mogri M, Pashaie R, Goo W, Yizhar O, Ramakrishnan C, Deisseroth K, Shenoy KV (2011) An optogenetic toolbox designed for primates. Nat Neurosci 14:387-397

Dong S, Rogan SC, Roth BL (2010) Directed molecular evolution of DREADDs: a generic approach to creating next-generation RASSLs. Nat Protoc 5:561-573

Ekstrom LB, Roelfsema PR, Arsenault JT, Bonmassar G, Vanduffel W (2008) Bottom-up dependent gating of frontal signals in early visual cortex. Science 321:414-417

Ekstrom LB, Roelfsema PR, Arsenault JT, Kolster H, Vanduffel W (2009) Modulation of the contrast response function by electrical microstimulation of the macaque frontal eye field. $\mathrm{J}$ Neurosci 29:10683-10694

Friston K (2011) Functional and effective connectivity: a review. Brain Connect 1:13-36

Gerits A, Vanduffel W (2013) Optogenetics in primates: a shining future? Trends Genet 29:403-411

Gerits A, Ruff CC, Guipponi O, Wenderoth N, Driver J, Vanduffel W (2011) Transcranial magnetic stimulation of macaque frontal eye fields decreases saccadic reaction time. Exp Brain Res 212:143-152

Gerits A, Farivar R, Rosen BR, Wald LL, Boyden ES, Vanduffel W (2012) Optogenetically induced behavioral and functional network changes in primates. Curr Biol 22:1722-1726

Han X, Qian X, Bernstein JG, Zhou HH, Franzesi GT, Stern P, Bronson RT, Graybiel AM, Desimone R, Boyden ES (2009) Millisecond-timescale optical control of neural dynamics in the nonhuman primate brain. Neuron 62:191-198

Hikosaka O, Wurtz RH (1985) Modification of saccadic eye movements by GABA-related substances. I. Effect of muscimol and bicuculline in monkey superior colliculus. J Neurophysiol 53:266-291

Histed MH, Bonin V, Reid RC (2009) Direct activation of sparse, distributed populations of cortical neurons by electrical microstimulation. Neuron 63:508-522

Janssens T, Keil B, Farivar R, McNab JA, Polimeni JR, Gerits A, Arsenault JT, Wald LL, Vanduffel W (2012) An implanted 8-channel array coil for high-resolution macaque MRI at 3T. Neuroimage 62:1529-1536 
Janssens T, Zhu Q, Popivanov ID, Vanduffel W (2014) Probabilistic and single-subject retinotopic maps reveal the topographic organization of face patches in the macaque cortex. J Neurosci 34:10156-10167

Jazayeri M, Lindbloom-Brown Z, Horwitz GD (2012) Saccadic eye movements evoked by optogenetic activation of primate V1. Nat Neurosci 15:1368-1370

Kinoshita M, Matsui R, Kato S, Hasegawa T, Kasahara H, Isa K, Watakabe A, Yamamori T, Nishimura Y, Alstermark B, Watanabe D, Kobayashi K, Isa T (2012) Genetic dissection of the circuit for hand dexterity in primates. Nature 487:235-238

Lomber SG, Cornwell P, Sun J-S, MacNeil MA, Payne BR (1994) Reversible inactivation of visual processing operations in middle suprasylvian cortex of the behaving cat. Proc Natl Acad Sci USA 91:2999-3003

Lomber SG, Payne BR, Horel JA (1999) The cryoloop: an adaptable reversible cooling deactivation method for behavioral or electrophysiological assessment of neural function. J Neurosci Methods 86:179-194

Markram H (2012) The human brain project. Sci Am 306:50-55

McAdams CJ, Maunsell JR (1999) Effects of attention on orientation-tuning functions of single neurons in macaque cortical area V4. J Neurosci 19:431-441

Moore T, Armstrong KM (2003) Selective gating of visual signals by microstimulation of frontal cortex. Nature 421:370-373

Moore T, Fallah M (2004) Microstimulation of the frontal eye field and its effects on covert spatial attention. J Neurophysiol 91:152-162

Noudoost B, Moore T (2011) Control of visual cortical signals by prefrontal dopamine. Nature 474:372-375

Ohayon S, Grimaldi P, Schweers N, Tsao DY (2013) Saccade modulation by optical and electrical stimulation in the macaque frontal eye field. J Neurosci 33:16684-16697

Okano H, Miyawaki A, Kasai K (2015) Brain/MINDS: brain-mapping project in Japan. Philos Trans R Soc Lond B Biol Sci 370

Packer AM, Roska B, Hausser M (2013) Targeting neurons and photons for optogenetics. Nat Neurosci 16:805-815

Premereur E, Vanduffel W, Roelfsema PR, Janssen P (2012) Frontal eye field microstimulation induces task-dependent gamma oscillations in the lateral intraparietal area. J Neurophysiol 108:1392-1402

Premereur E, Vanduffel W, Janssen P (2014) The effect of FEF microstimulation on the responses of neurons in the lateral intraparietal area. J Cogn Neurosci 26(8):1672-1684

Rasmussen T, Penfield W (1947) The human sensorimotor cortex as studied by electrical stimulation. Fed Proc 6:184

Reynolds JH, Heeger DJ (2009) The normalization model of attention. Neuron 61:168-185

Schall JD (1997) Visuomotor areas of the frontal lobe. In: Rockland K (ed) Cerebral Cortex. Plenum Press, New York, pp 527-638

Schall JD, Morel A, King DJ, Bullier J (1995) Topography of visual cortex connections with frontal eye field in macaque: convergence and segregation of processing streams. J Neurosci 15:4464-4487

Tehovnik EJ, Lee K (1993) The dorsomedial frontal cortex of the rhesus monkey: topographic representation of saccades evoked by electrical stimulation. Exp Brain Res 96:430-442

Tufail Y, Matyushov A, Baldwin N, Tauchmann ML, Georges J, Yoshihiro A, Tillery SI, Tyler WJ (2010) Transcranial pulsed ultrasound stimulates intact brain circuits. Neuron 66:681-694

Van Essen DC et al (2012) The Human Connectome Project: a data acquisition perspective. Neuroimage 62:2222-2231

Vanduffel W, Payne BR, Lomber SG, Orban GA (1997) Functional impact of cerebral connections. Proc Natl Acad Sci USA 94:7617-7620

Vanduffel W, Zhu Q, Orban GA (2014) Monkey cortex through fMRI glasses. Neuron 83:533-550

Yizhar O, Fenno LE, Prigge M, Schneider F, Davidson TJ, O'Shea DJ, Sohal VS, Goshen I, Finkelstein J, Paz JT, Stehfest K, Fudim R, Ramakrishnan C, Huguenard JR, Hegemann P, Deisseroth K (2011) Neocortical excitation/inhibition balance in information processing and social dysfunction. Nature 477:171-178 